\title{
Multi-area Environmental Economic Dispatch with Reserve Constraints Using Enhanced Particle Swarm Optimization
}

\author{
Vinay Kumar Jadoun ${ }^{\mathrm{a}}$, Nikhil Gupta ${ }^{\mathrm{a}}$, Khaleequr Rehman Niazi ${ }^{\mathrm{a}}$, Anil Swarnkar ${ }^{\mathrm{a}}$ \& \\ Ramesh C. Bansal ${ }^{b^{*}}$ \\ ${ }^{a}$ Malaviya National Institute of Technology, Jaipur, India \\ ${ }^{\mathrm{b}}$ Department of Electrical, Electronic and Computer Engineering, University of Pretoria, \\ Pretoria, South Africa
}

\begin{abstract}
In this paper, the multi-area environmental economic dispatch (MAEED) problem with reserve constraints is solved by proposing an enhanced particle swarm optimization (EPSO) method. The objective of MAEED problem is to determine the optimal generating schedule of thermal units and inter-area power transactions in such a way that total fuel cost and emission are simultaneously optimized while satisfying tie-line, reserve, and other operational constraints. The spinning reserve requirements for reserve-sharing provisions are investigated by considering contingency and pooling spinning reserves. The control equation of the particle swarm optimization (PSO) is modified by improving the cognitive component of the particle's velocity using a new concept of a preceding experience. In addition, the operators of PSO are dynamically controlled to maintain a better balance between cognitive and social behavior of the swarm. The effectiveness of the proposed EPSO has been investigated on four areas, 16 generators and four areas, 40 generators test systems. The application results show that EPSO is very promising to solve the MAEED problem.
\end{abstract}

Keywords : contingency spinning reserve, multi-area economic-emission dispatch, particle swarm optimization, pooling spinning reserve, tie-line capacity

\section{Introduction}

Modern power systems are large, with multiple control areas interconnected through tie-lines. Each control area has its own load and generation. Areas of individual utility are interconnected through tie-lines to operate with maximum reliability, reserve sharing, improved security, and less production cost than when operated as an isolated area [1]. In an interconnected power system, several generation companies join to form a power pool with an aim to gain economic benefits in their operations. The benefits of the pool depend on several factors such as the characteristics of a pool, types of interconnections, tie-line limits, and spinning reserve requirements. The regulatory bodies have enforced certain norms to keep a definite amount of generation in each area as a contingency spinning reserve to meet its own contingencies and a definite amount of generation in the area as pooling reserve. The economic benefit of the pool also depends on how the pooling reserve is handled. Moreover, regulatory bodies have also imposed limits and penalties on the emission of pollutants due to environmental concerns. Therefore, multi-area economic dispatch (MAED) with the consideration of emission, transmission capacity constraint, and reserve sharing is more practical in the context of modern power systems.

Some early efforts to attempt the MAED problem can be briefly stated as follows: Sharma et al. [2] formulated the MAED problem with area spinning reserve constraints and without 
the consideration of emission. They established that more economy can be achieved using reserve sharing. Shoults et al. [3] suggested import and export constraints between areas that can be analyzed on a daily, monthly, and annual basis. Romano et al. [4] formulated the economic dispatch problem with line flow constraints and spinning reserves using the linear optimization program and solved using the Dantzig-Wolfe decomposition principle. Desell et al. [5] described an application of linear programming to transmission-constrained generation production cost analysis in power system planning. Wang and Shahidehpour [6] proposed a decomposition approach to non-linear multi-area generation scheduling with tieline constraints using expert systems. The authors showed the efficiency of their proposed approach by testing it on a four-area system, with each area consisting of 26 units [7]. In recent years, modern artificial intelligence-based techniques have shown potential to solve such complex combinatorial constrained optimization problems due to their ability to obtain global or near-global optima. Jayabarathi et al. [7] solved MAED problems with tie-line constraints using evolutionary programming. Chen and Chen [8] presented direct search method for solving economic dispatch problem considering transmission capacity constraints. Manoharan et al. [9] proposed covariance matrix-adapted evolutionary strategy for a multiarea dispatch where a Karush Kuhun Tucker (KKT) optimality criterion is applied to guarantee the optimal convergence. Zhu [10] presented a new non-linear optimization neural network approach to study security-constrained interconnected multi-area dispatch problems. Basu [11] employed artificial bee colony optimization with a variety of system constraints. However, effluent emissions from thermal units have not been given serious consideration by these attempts. In the recent past, the optimal multi-area environmental economic dispatch (MAEED) problem has been attempted by Wang and Singh [12]. They formulated the problem by considering tie-line transfer capacities and area spinning reserve sharing to ensure security and improved reliability, respectively. But the norms of regulatory bodies enforce utilities to keep a definite amount of reserve, i.e., contingency spinning reserve, in each area to meet out their own contingencies. From the literature survey, it is found that MAED with the consideration of emission, tie-line constraints, and spinning reserve requirement has not been attempted.

In the light of the above discussion, a new formulation of economic emission dispatch is proposed in this paper by extending the economic dispatch problem to an MAEED problem with the consideration of transmission capacity constraints and with a new reserve sharing approach. This formulation increases the complexity of the dispatch problem that arises due to conflicting nature of cost and emission objectives, stringent area power balance constraints, tie-line constraints, and area spinning reserve constraints, in addition to the other operational constraints [2]. The proposed formulation is solved using an improved particle swarm optimization (PSO)-based method proposed in this paper. A new concept is also proposed to share spinning reserve requirement, which results in substantial saving in overall spinning reserve requirement.

PSO is a swarm intelligence-based meta-heuristic optimization technique that has shown its proven potential to solve diverse engineering optimization problems. Researchers are attracted toward this technique due to its simplicity, convergence speed, and robustness. However, PSO has an inherent tendency of local trapping. Several modified versions of PSO have been reported in the recent past to enhance its performance by modulating inertia weight [13-17], improving cognitive and social behavior [14, 17-20], using constriction factor approach [21, 22], modifying the control equation of the PSO [13, 16, 20, 23-27], or squeezing the search space $[26,27]$, etc. However, some of these versions of PSO require exhaustive experimentations for parameter setting and some additional mechanism to avoid 
local trapping. Some others employed empirical formulae to regulate particle's velocity in order to maintain a better balance between cognitive and social behavior of the swarm.

In this paper, an improved variant of PSO, i.e., EPSO, is proposed by exponentially varying inertia weight, cognitive, and social components of particles' velocities in such a way that a proper balance is maintained between cognitive and social behaviors of the swarm throughout the computational process. For this purpose, the concept of preceding experience and exponentially varying constriction functions are suggested, which are not yet reported in the literature. A constraint handling algorithm is also proposed especially to deal with inter-area constraints related to inter-exchange of power, tie-line constraints, and spinning reserve requirements. A new scheme for inter-area spinning reserve sharing is proposed to cater contingency reserve requirements of each area.

The proposed method effectively regulates the velocity of particles during their flights so as to enhance its exploration and exploitation potentials. The economic and environmental objectives of ED problem are combined in a fuzzy framework to solve this multi-objective optimization problem. The effectiveness of the proposed method has been investigated on two four-areas test systems of different dimensions considering various operational constraints such as valve-point loading effects, power balance, tie-line capacity, and spinning reserves. The performance of the proposed method is compared with other established methods.

\section{Problem Formulation}

A large interconnected power system is generally composed of different areas or zones based on various criteria such as geographical, operational, planning, and organizational. Each of these areas is interconnected to its neighboring areas through tie-lines. Each area has its own generation, load, and spinning reserve. These areas were planned for exchange of operational surpluses among different control areas. From a market point of view, these areas are decentralized. However, for technical reasons, there is one independent system operator (ISO) that imposes certain operational restrictions on these control areas for the purpose of grid security such as tie-line constraints and total spinning reserve constraints. In a deregulated power system, generally different power generation companies of different areas pool together with the objective to achieve the most economical generation policy that could supply the local demands without violating certain operational constraints imposed by the ISO. The proposed approach assumed that there is pooling of different generation companies of different areas to achieve the common goal of maximizing their profits. The aim of the MAEED is therefore to dispatch the generators of a power pool for the forecasted load in such a fashion that optimizes the fuel cost and pollutant emissions from thermal units while satisfying operational constraints, contingency reserve constraints, and transmission capacity constraints. In the proposed formulation, the non-contingency reserved is shared. The mathematical formulation of MAEED is described further.

\subsection{Generator Fuel Cost Function}

The generator cost function is generally considered as quadratic when valve-point effects are neglected. However, large turbine generators usually have a number of fuel admission valves that are operated in sequence to meet out the increased generation. The opening of a valve increases the throttling losses rapidly and thus the incremental heat rate rises suddenly. This valve-point effect introduces ripples in the heat-rate curves and can be modeled as sinusoidal 
function in the cost function. Therefore, the objective function for the MAED problem may be stated as to minimize the following:

$$
\begin{aligned}
F\left(P_{G m j}\right)= & \sum_{m=1}^{M} \sum_{j=1}^{N_{G m}}\left(a_{m j}+b_{m j} P_{G m j}+c_{m j} P_{G m j}^{2}\right) \\
& +\left|e_{m j} \sin \left(f_{m j}\left(P_{G m j}^{\mathrm{min}}-P_{G m j}\right)\right)\right|
\end{aligned}
$$

where $a_{m j}, b_{m j}, c_{m j}$ are the cost coefficients, and $e_{m j}$ and $f_{m j}$ are the valve point effect coefficients of the $j$ th generator in area $m, P_{G m j}$ is the real power output of the $j$ th generator in area $m, P_{G m j}^{\min }$ is the minimum generation limit of the $j$ th generator in area $m, M$ is the number of areas, and $N_{G m}$ is the number of generating units in the system in area $m$.

\subsection{Pollutant Emission Function}

The pollutant emission produced by thermal plants can be expressed as a sum of a quadratic and an exponential function as follows:

$E\left(P_{G m j}\right)=\sum_{m=1}^{M} \sum_{j=1}^{N_{G m}} \alpha_{m j}+\beta_{m j} P_{G m j}+\gamma_{m j} P_{G m j}^{2}$

where, $\alpha_{m j}, \beta_{m j}$, and $\gamma_{m j}$ are the emission coefficient of the $j$ th generator in area $m$.

Subject to the following constraints.

\subsection{Power Balance Constraints}

In area $m$, the total power generation of all generators must be equal to the area power demand $P_{D m}$ with the consideration of imported and exported power [1] and can be stated as follows:

$$
\sum_{j=1}^{N_{G m}} P_{G m j}=P_{D m}+\sum_{k, k \neq m} P_{T m k} ; m \in M
$$

where $P_{D m}$ is the power demand of area $m ; P_{T m k}$ is the tie-line real power transfer from area $m$ to area $k . P_{T m k}$ is positive when power flows from area $m$ to area $k$ and is negative when power flows from area $k$ to area $m$.

\subsection{Generator Constraints}

For stable operation, power output of each generator is restricted within its minimum and maximum limits. The generator power limits are expressed as follows:

$$
P_{G m j}^{\min } \leq P_{G m j} \leq P_{G m j}^{\max }
$$

where $P^{\min }{ }_{G m j}$ and $P_{G m j}{ }^{\max }$ are the minimum and maximum generation limits of the $j$ th generator in area $m$. 


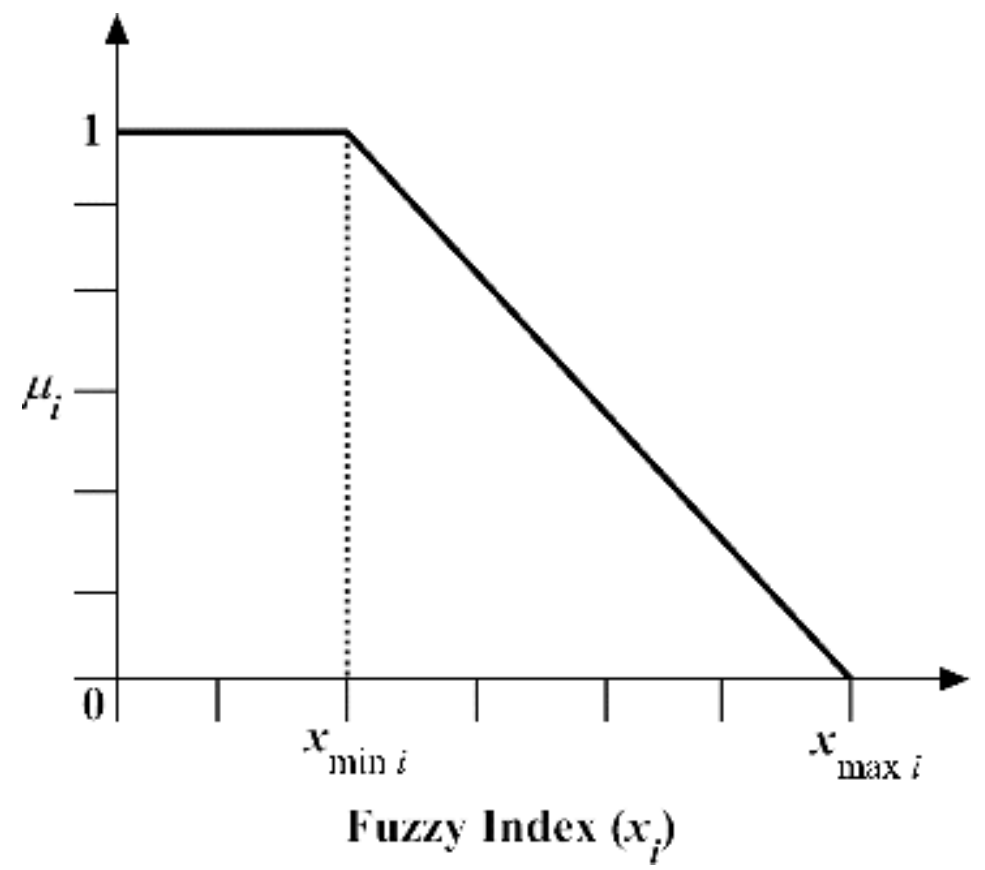

Figure 1 The conventional trapezoidal fuzzy membership function.

\subsection{Tie-line Capacity Constraints}

The transfer of real tie-line power $P_{T m k}$ from area $m$ to area $k$ should not exceed the maximum tie-line limit for security consideration and it is expressed as below:

$-P_{T m k}^{\max } \leq P_{T m k} \leq P_{T m k}^{\max }$

where $P^{\max }{ }_{T m k}$ is the maximum tie-line power limit from area $m$ to area $k$.

\subsection{Area Spinning Reserve Constraints}

In a power pool, generally a fixed reserve is kept in each area to meet the contingency requirement of that area. This reserve may be called as contingency spinning reserve of the area. In addition, a pooling spinning reserve is also kept to meet the emergency requirement of the power pool such as loss of generation in any area of the pool. This pooling spinning reserve can either be kept in one area as a supplementary reserve or it may be contributed by multiple areas of the pool. When this reserve is kept in each area, the total specified spinning reserve in each area is the sum of contingency reserve and supplementary reserve of that area. In Ref. [12], the contingency reserve of an area and contribution of that area to pool reserve are combined and termed as specified/required spinning reserve of that area. However, if only contingency reserve of an area is kept as specified reserve of that area and the pool reserve is shared among all areas of the pool, it may result in less spinning reserve requirement in each area and thereby reducing the overall reserve requirement of the pool. It is therefore proposed that the spinning reserve requirement of an area $i$ should satisfy the following equation:

$\sum_{j=1}^{N_{G m}} S_{m j} \geq S_{c m}+S_{p m}+\sum_{k, k \neq m} R C_{m k}$

where $S_{m j}$ is the available reserve on the $j$ th unit of $m$ th area, $S_{c m}$ is the contingency spinning reserve in the $m$ th area, $S_{p m}$ is the pooling spinning reserve in the $m$ th area, and $R C_{m k}$ is the pool reserve contributed from area $m$ to area $k$. 


\subsection{Multi-objective Formulation in Fuzzy Framework}

In fuzzy domain, each objective is associated with a membership function. The membership function indicates the degree of satisfaction of the objective. The trapezoidal fuzzy function, as shown in Figure 1, provides a linear and continuous relationship between the fuzzy membership function and the fuzzy index of the concern objective and assigns any membership value between 0 and 1 to the objectives. The conventional trapezoidal fuzzy membership function [28-31] is used to combine various objectives.

Mathematically,

$$
\mu_{i}=\left\{\begin{array}{l}
1 ; x_{i} \leq x_{\min i} \\
M x_{i}+C ; x_{\min i} \leq x_{i} \leq x_{\max i} \\
0 ; x_{i} \geq x_{\max i}
\end{array}\right.
$$

The lower and upper bounds of the desired objective are $x_{\min i}$ and $x_{\max i}$, respectively, and can be varied according to the preferences of different operators. If $x_{i} \leq x_{\min i}$, a unity membership value and if $x_{i} \geq x_{\max i}$, a zero membership value is assigned. The coefficients $M$ and $C$ are decided by the lower and upper bounds of the fuzzy index $x_{i}$ and are given by

$$
\begin{aligned}
M & =-1 /\left(x_{\max i}-x_{\min i}\right) \\
C & =x_{\max i} /\left(x_{\max i}-x_{\min i}\right) \\
C & =x_{\max i} /\left(x_{\max i}-x_{\min i}\right)
\end{aligned}
$$

Now a single objective function can be used to solve this MAEED problem as to

$\operatorname{Max} \mu=\left(\mu_{1} \mu_{2}\right)^{1 / 2}$

where $\mu_{1}$ and $\mu_{2}$ denote fuzzy membership functions for the fuel cost and pollutant emission, respectively, and $\mu$ is the overall fuzzy membership function for two objectives.

Subject to the generator constraints defined by (3)-(6).

\section{Proposed EPSO}

The conventional PSO is initialized with a population of random solutions and searches for optima by updating particle positions. The velocity of the particle is influenced by three components, namely, initial, cognitive, and social components. Each particle updates its previous velocity and position vectors according to the following model of [32].

$$
\begin{aligned}
v_{i}^{k+1}= & W v_{i}^{k}+C_{1} \times \text { rand }_{1}() \times \frac{\text { pbest }_{i}^{k}-s_{i}^{k}}{\Delta t} \\
& +C_{2} \times \operatorname{rand}_{2}() \times \frac{\text { gbest }^{k}-s_{i}^{k}}{\Delta t} \\
s_{i}^{k+1}= & s_{i}^{k}+v_{i}^{k+1} \times \Delta t \\
& +C_{2} \times \operatorname{rand}_{2}() \times \frac{\text { gbest }^{k}-s_{i}^{k}}{\Delta t} \\
s_{i}^{k+1}= & s_{i}^{k}+v_{i}^{k+1} \times \Delta t
\end{aligned}
$$

where $v_{i k}$ is the velocity of $i$ th particle at $k$ th iteration, $\operatorname{rand}_{1}()$ and $\operatorname{rand}_{2}()$ are random numbers between 0 and $1, s_{i}{ }^{k}$ is the position of $i$ th particle at $k$ th iteration, $C_{1}, C_{2}$ are the acceleration coefficients, pbest $_{i}{ }^{k}$ is the best position of $i$ th particle achieved based on its own experience, gbest $^{k}$ is the best particle position based on overall swarm experience, $\Delta t$ is the 
time step, usually set to $1 \mathrm{sec}$, and $W$ is the inertia weight and is allowed to decrease linearly as follows:

$W=W_{\min }+\frac{\left(W_{\max }-W_{\min }\right) \times\left(i t r_{\max }-i t r\right)}{i t r_{\max }}$

where $W_{\min }$ and $W_{\max }$ are the respective minimum and maximum bounds of the inertia weight, $i t r_{\max }$ is the maximum number of iterations, and itr is the current iteration.

For better performance of PSO, the particles must fly with higher velocities during the early flights to enhance global search and should gradually slow down during later flights of the journey to improve the local search. Therefore, with appropriate regulation of particle's velocity, the performance of PSO can be improved. This requires a proper balance between cognitive and social behaviors of the swarm. Initially, the cognitive component must dominate over the social component to ensure global exploration of the search space. However, during the later part of the journey, the social component must dominate over the cognitive one so as to divert all particles toward the global best to improve the local exploitation. This is essential for a good balance between exploration and exploitation as suggested by [10].

In the conventional PSO, only the initial velocity component is regulated by inertia weight. However, the cognitive and social behavior of the swarm, though randomized to ensure diversity, is statically controlled by assigning constant values to acceleration coefficients. These cognitive and social components of velocity are added in the regulated initial velocity component to decide the movement of particles. This probably causes uncontrolled particle velocities during the whole computation process and thus results in insufficient exploration and exploitation of the search space. As a consequence, the conventional PSO inherently exhibits poor convergence due to local trapping. Therefore, a modified control equation is suggested for dynamically regulating particle's velocity by suggesting suitable exponential constriction functions $\zeta_{1}$ and $\zeta_{2}$. Moreover, the cognitive and social components are modified by considering the preceding experience. The suggested control equation for the proposed EPSO may be expressed as

$$
\begin{aligned}
v_{i}^{k+1}= & W \times v_{i}^{k}+\zeta_{1} \times C_{1 b} \times \text { rand }_{1}() \times \frac{\text { pbest }_{i}^{k}-s_{i}^{k}}{\Delta t} \\
& +\left(1-\zeta_{1}\right) \times C_{1 p} \times \text { rand }_{2}() \times \frac{s_{i}^{k}-\text { ppreceding }_{i}^{k}}{\Delta t} \\
& +\zeta_{2} \times C_{2} \times \text { rand }_{3}() \times \frac{\text { gbest }^{k}-s_{i}^{k}}{\Delta t}
\end{aligned}
$$

where $C_{l b}$ and $C_{l p}$ are acceleration coefficients representing cognitive behavior for the best and preceding experiences and ppreceding ${ }_{i}{ }^{k}$ is the preceding position of the $i$ th particle for the $k$ th iteration. In the proposed method, the inertia weight is modified to regulate the tradeoff between the global exploration and the local exploitation of the swarm. The preceding experience has been added to improve the cognitive component. Further, dynamic acceleration coefficients have been introduced using constriction functions to regulate the cognitive and social behaviors of the swarm. These proposed modifications are discussed in the following sections. 


\subsection{Inertia Weight Update}

The trend of linear modulation of inertia weight of [33] is followed to solve optimization problems using PSO by many researchers till date [23, 24, 34, 35]. For large-scale optimization problems, there exist a large number of local optima in the close vicinity of the global optima. Therefore, the exploitation potential of the search algorithm should be sufficient to obtain better solutions. Therefore, the inertia weight has been intuitively varied exponentially with respect to iterations. Modulations suggested to update the inertia weight is governed by the following relation:

$$
W=\exp \left(-\eta \log _{\mathrm{e}}\left(W_{\max } / W_{\min }\right)\right) ; \eta=i t r / i t r_{\max }
$$

\subsection{Updating Preceding Experience}

The cognitive behavior was split in [24] by considering also the worst experience in addition to the best experience of the particle to provide some additional diversity, but it results in poor local exploitation unless supported by a local random search. Therefore, the concept of the preceding experience is suggested where the current fitness of each particle is compared with its fitness value in the preceding iteration, and if it is found less, it will be treated as the preceding experience. The preceding experience of the particle produces much less diversity than the worst experience and thus can provide better exploration and exploitation of the search space without employing any additional local random search or else.

\subsection{Dynamic Control of Acceleration Coefficients}

The cognitive and social behaviors introduced in the conventional PSO play an important role in searching the promising area where the global optima may exist and thereafter approaching toward the global optima. In conventional PSO, these behaviors are governed by static acceleration coefficients. However, many researchers [14, 17-20, 36] suggested that these acceleration coefficients must be dynamically controlled with iterations to regulate particle's velocity during the whole computation process. In the present work, following the logic of dynamic inertia weight, the acceleration coefficients are dynamically controlled by introducing two exponential constriction functions $\zeta_{1}$ and $\zeta_{2}$. These constriction functions dynamically regulate the cognitive and social behaviors of the swarm, thus limiting particles' velocities during their whole course of the flight and are proposed as

$$
\begin{aligned}
& \zeta_{1}=\exp (-\mu \eta) \\
& \zeta_{2}=k_{c} \exp (\mu \eta)
\end{aligned}
$$

The constriction functions help to maintain the dynamic behaviors of cognitive and social components. In the beginning, the cognitive component dominates over the social one. As the iterations proceed, the cognitive component drops sharply, whereas the social component builds up gradually. After a certain iteration count, they attain same values. Let these components become equal when $\eta$ is $\eta_{t}$, and then the constriction factor $k_{\mathrm{c}}$ is given by

$$
\left.k_{\mathrm{c}}=C_{1 b} \exp \left(-2 \mu \eta_{t}\right)\right) / C_{2}
$$

Beyond $\eta_{t}$, the social component dominates over the cognitive component till the end of the search. Let it become $k_{s}$ when the iteration count is exhausted, and is given by 
$k_{\mathrm{s}}=k_{\mathrm{c}} C_{2} \exp (\mu)$

From (18) and (19)

$k_{\mathrm{s}}=C_{1 b} \exp \left(\mu\left(1-2 \eta_{t}\right)\right)$

Interestingly, $k_{\mathrm{s}}$ is independent of $C_{2}$, but it is the function of $C_{1 b}$. In the light of [16], $k_{\mathrm{s}}$ cannot be more than $C_{1 b}$ and thus $\eta_{t}$ should be more than 0.5 . Further, the cognitive component will not be perceptible for $\mu$ to be five or more [37]. The coefficients of exponents terms $\mu$ and the constriction factor $k_{\mathrm{c}}$ dynamically govern the cognitive and social components of particles' velocities. Therefore, both are responsible to maintain a proper balance between cognitive and social behavior of the swarm during the whole course of the flight. For the given values of static acceleration coefficients, both $\mu$ and $\eta_{t}$ can be optimized to determine the optimal value of $k_{\mathrm{s}}$ using (20) and then the optimal value of $k_{\mathrm{c}}$ can be obtained using (18). These alterations in the control equation of the conventional PSO regulates particles' velocities without any additional formulation as reported in many improved versions of PSO [19, 27, 38, 39], yet preserving diversity due to the stochastic nature of cognitive and social behaviors of the swarm.

\subsection{Particle Encoding and Initialization}

The solution of an MAED problem is the set of the most optimal generations and the connected tie-lines of that area for the desired objective (s) bounded by certain operational constraints. In the proposed PSO, the particles are encoded in real numbers as the set of current generations and the connected tie-lines of that area in MW, as shown in Figure 2.

$$
\begin{aligned}
& P_{G / l} P_{G l 2} \quad \cdots \quad P_{G l j} \cdots \cdots \quad \ldots . \quad P_{G l N}
\end{aligned}
$$

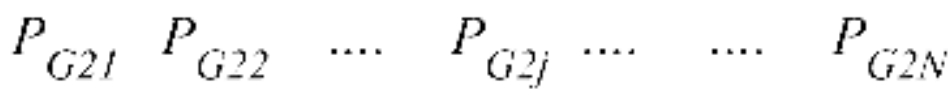

$$
\begin{aligned}
& P_{G m 1} P_{G m 2}, \ldots P_{G m j} \cdots \quad \ldots \quad P_{G m n} \\
& P_{T l 2} \quad P_{T / 3} \quad \cdots \quad P_{T 23} \cdots \cdots \quad \cdots, P_{T m k}
\end{aligned}
$$

Figure 2 Particle encoding for the proposed EPSO.

The initial population is randomly created with predefined number of particles to maintain diversity. Each of these particles satisfies the problem constraints defined by (3)-(6). Infeasible particles, whenever appeared, are corrected employing a constrained handling algorithm as described later in the section. The fitness of each particle is evaluated using (10) and then pbest, ppreceding, and gbest are initialized. The initial velocity of particles is assumed to be zero. 


\subsection{Constrained Handling}

The velocity and position update may create infeasible solutions. In the proposed method, infeasible individuals are not rejected but are corrected to feasible ones using a constrained handling algorithm. For this purpose, the generations of all generators are adjusted by their respective bounded generation limits, tie-line, and area spinning reserve constraints as given in Eqs. (3)-(6). If the generations are lesser or greater than minimum or maximum generation level, then the corresponding generation is set at minimum or maximum bound limits as in (4). Similarly, if the transfer of real tie-line power from area $i$ to area $k$ exceeds its limit, then the corresponding tie-line power is set at tie-line bound limits as in (5) for security consideration. For area spinning reserve constraint, every area has to fulfill its respective reserve requirement as per (6), and if not satisfied, then the difference amount of reserve is distributed equally among all generating units and the connected tie-lines of that area till it is satisfied. The power balance error is calculated using (3). The error in the power is also equally distributed among all generators and the procedure is repeated till the error is reduced to a predefined mismatch value, say $0.001 \mathrm{MW}$.

\subsection{Elitism and Termination Criterion}

In stochastic-based algorithms like PSO, the solution with the best fitness in the current iteration may be lost in the next iteration. Therefore, the particle with the best fitness is kept preserved for the next iteration. The algorithm is terminated when either all particles reach to the global best position or the predefined maximum iteration number is reached.

\section{Simulation Results and Discussion}

The proposed algorithm is tested on two test systems, i.e., four areas, 16 generators system and four areas 40 generators system. The value of acceleration coefficients $C_{1 b}, C_{1 p}$, and $C_{2}$ for these test systems are taken as 1.6, 0.4, and 2.0, respectively, as in [24]. The value of maximum and minimum bounds of the inertia weight is taken as 0.9 and 0.1 , respectively. The swarm size and maximum iteration count have been obtained after usual tradeoff. A swarm size of 20 and 100 is taken for these test systems, respectively, and the maximum iteration count is taken as 1000 and 2500 . The proposed algorithm has been developed using MATLAB and the simulations have been carried on a personal computer of Intel i5, 3.2 GHz, and 4 GB RAM.

To determine the optimal value of $k_{\mathrm{s}}$, several experimentations have been performed by varying $\mu$ and $\eta_{t}$ in the expected range $[5,5.5]$ and $[0.5,0.75]$ for test system 1 . The results obtained for MAED on the basis of average fuel cost and its standard deviation (STD) after100 independent trials of EPSO are presented in Table 1. The table shows that the optimal value of $k_{\mathrm{s}}$ is 0.2 and the corresponding values of $\mu$ and $\eta_{t}$ are found to be 5.2 and 0.70 , respectively. The proposed EPSO is now applied on each test systems with following three different cases. 
Table 1 Optimizing $k_{\mathrm{s}}$

\begin{tabular}{|l|l|l|}
\hline $\boldsymbol{k}_{s}$ & Average fuel cost $\mathbf{( \$ / h r )}$ & STD \\
\hline 0.1 & 2144.714787 & 0.006806 \\
\hline 0.2 & 2143.824856 & 0.006575 \\
\hline 0.3 & 2144.749566 & 0.006837 \\
\hline 0.4 & 2146.536661 & 0.006939 \\
\hline 0.5 & 2152.244788 & 0.008060 \\
\hline
\end{tabular}

1. Case 1: Without inter-area aid

2. Case 2: Inter-area aid with reserve sharing

3. Case 3: Inter-area aid with proposed reserve sharing

In case 1, all areas are assumed not be interconnected by tie-lines and every area has to individually satisfy its own reserve requirement. In case 2, the areas are interconnected and individual area reserves are mutually shared, whereas in case 3, the areas are interconnected and reserve sharing is allowed while keeping intact the contingency and pooling spinning reserve constraints. The application results obtained by the proposed PSO after 100 independent trials are presented and compared with other existing population-based techniques.

\subsection{Test System 1}

This system consists of four areas, each with four thermal units and the transmission losses are neglected. All the four areas are interconnected through six tie-lines as shown in Figure 3. The detailed data of this system may be referred from [12]. The system base MVA is considered as $100 \mathrm{MVA}$. The area power demand is $0.3,0.5,0.4$, and 0.6 p.u., respectively. The minimum and maximum limits of tie-lines are considered as in [12].

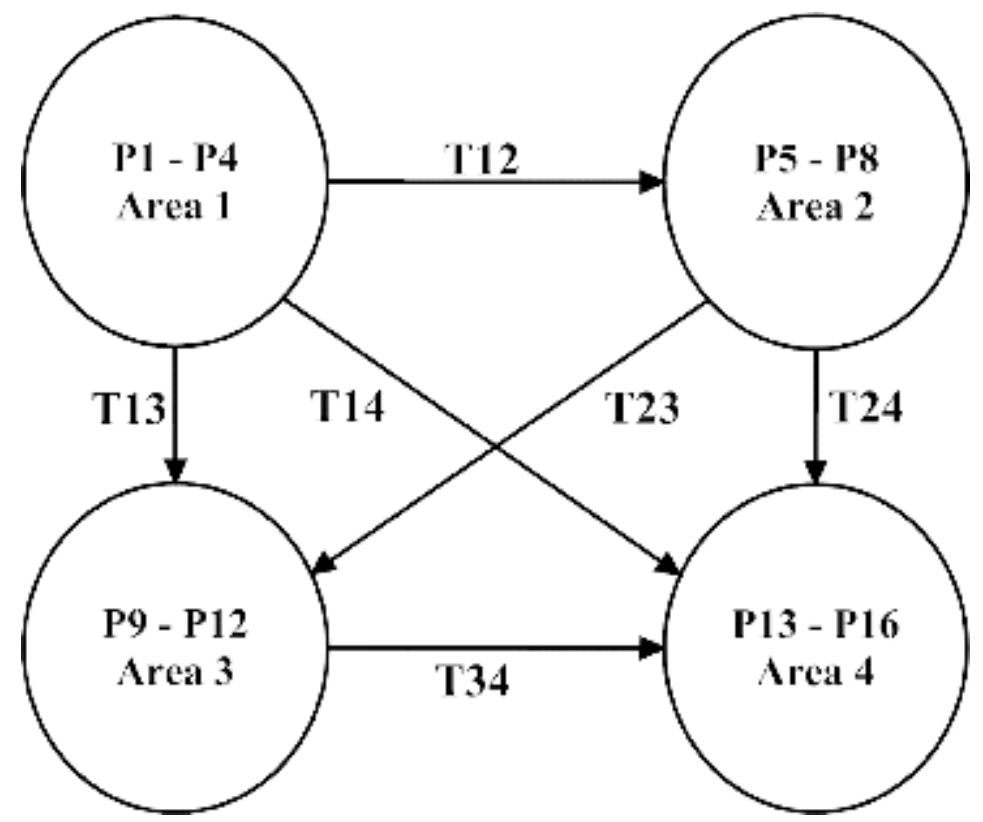

Figure 3 Four areas 16 generators system for test system 1. 
For case 1, the value of specified spinning reserve of each area is $30 \%$ of area power demand as in [12]. To show the effectiveness of the proposed EPSO, it is applied for MAED of this system. The minimum fuel cost obtained using EPSO is found to be $2143.824 \$ / \mathrm{hr}$, which is better than $2181.261 \$ / \mathrm{hr}$, obtained by the differential evolution with chaotic sequences (DEC2) of [2]. The proposed method is then applied for MAEED problem for this system. The optimal fuel cost and emission obtained are $2172.522 \$ / \mathrm{hr}$ and $2.997 \mathrm{ton} / \mathrm{hr}$, respectively. The method of [12] provides Pareto solutions for this system with fuel cost variation from $2191.140 \$ / \mathrm{hr}$ to $2191.270 \$ / \mathrm{hr}$ and corresponding emission variation from 3.749 ton $/ \mathrm{hr}$ to $3.692 \mathrm{ton} / \mathrm{hr}$. From this result, it is clear that the proposed method provides a much better solution for both the objectives in comparison to the existing method [12]. Thus, the proposed EPSO has found a solution that dominates over the Pareto front generated by the MOPSO of [12]. This verifies the effectiveness of the proposed EPSO.

For case 2, the specified spinning reserve requirement for each area is taken same as in case 1. The MAEED problem is solved using EPSO. The best solution obtained provides a fuel cost and emission as $2165.7987 \$ / \mathrm{hr}$ and $2.8329 \mathrm{ton} / \mathrm{hr}$, respectively. The Pareto optimal solutions obtained by [12] shows extreme end solutions for fuel cost as $2166.8200 \$ / \mathrm{hr}$ and $2178.2000 \$ / \mathrm{hr}$. The corresponding emissions are obtained as $3.3152 \mathrm{ton} / \mathrm{hr}$ and $3.2301 \mathrm{ton} / \mathrm{hr}$, respectively. Thus, the proposed method provides a better solution than the existing method [12]. It may also be observed that the fuel cost is reduced from $2172.522 \$ / \mathrm{hr}$ to $2165.7987 \mathrm{\$} / \mathrm{hr}$ and the emission is reduced from $2.997 \mathrm{ton} / \mathrm{hr}$ to $2.8329 \mathrm{ton} / \mathrm{hr}$ when interarea flow of power is allowed.

For case 3, the proposed inter-area reserve sharing is employed by considering the contingency spinning reserve in each area, and the pooling reserve is contributed by all areas. The contingency spinning reserve for each area is taken as $7 \%$ of its power demand and the pooling spinning reserve is taken as $30 \%$ of the power demand of area 4 , having the highest loading. For this case, the proposed method provides fuel cost and emission as 2164.8558 $\$ / \mathrm{hr}$ and $2.4304 \mathrm{ton} / \mathrm{hr}$, respectively, which are less than that obtained in case 2 . This result highlights the importance of reserve sharing as proposed in this paper. Since this reserve sharing approach is new, no comparison results are available in the literature.

Table 2 Contingency, pooling spinning, and available reserves for case 3

\begin{tabular}{|c|c|c|c|c|}
\hline Reserve & Area 1 & Area 2 & Area 3 & Area 4 \\
\hline Contingency spinning reserve (p.u.) & 0.0210 & 0.0350 & 0.0280 & 0.0420 \\
\hline Pooling spinning reserve (p.u.) & \multicolumn{4}{|c|}{0.1800} \\
\hline Available reserve (p.u.) & 0.0618 & 0.1940 & 0.7938 & 0.5067 \\
\hline
\end{tabular}

Table 2 provides a quick reference to check the validity of the reserve sharing constraints imposed for the solution obtained. The table shows that the available reserve for each area is more than its respective contingency spinning reserve requirement. It can also be seen from the table that the sum of available reserves is maintained higher than the sum of contingency and pooling spinning reserve requirement. It can also be observed from the table that the total reserve to be maintained in case 3 is 0.306 p.u., whereas in case 2 it was 0.54 p.u. Thus, the proposed inter-area reserve sharing scheme provides significant reduction in the spinning reserve requirements. The optimal generating schedule and the corresponding tie-line flows obtained by the proposed method for MAEED may be referred from Table 3. It can be observed from the table that the optimal solution satisfied all the problem constraints. 
Table 3 Optimal generation schedule for test system 1

\begin{tabular}{|c|c|c|c|c|c|}
\hline & Case 1 & & Case 2 & & Case 3 \\
\hline Unit |l & Power (MW) & Unit & Power (MW) & Unit & Power (MW) \\
\hline 1,1 & 0.128330 & 1,1 & 0.120000 & 1,1 & 0.119800 \\
\hline 1,2 & 0.100000 & 1,2 & 0.060000 & 1,2 & 0.098700 \\
\hline $1,3[$ [ & 0.025700 & 1,3 & 0.110000 & 1,3 & 0.089900 \\
\hline 1,4 & 0.045876 & 1,4 & 0.110000 & 1,4 & 0.119900 \\
\hline 2,1 & 0.159713 & 2,1 & 0.188600 & 2,1 & 0.169500 \\
\hline 2,2 & 0.120000 & 2,2 & 0.117600 & 2,2 & 0.116700 \\
\hline 2,3 & 0.108913 & 2,3 & 0.132400 & 2,3 & 0.129100 \\
\hline 2,4 & 0.111280 & 2,4 & 0.103600 & 2,4 & 0.140700 \\
\hline $3,1 \quad[$ & 0.085696 & 3,1 & 0.066000 & 3,1 & 0.089900 \\
\hline 3,2 & 0.079593 & 3,2 & 0.063700 & 3,2 & 0.076100 \\
\hline 3,3$]$ & 0.085660 & 3,3 & 0.080900 & 3,3 & 0.101200 \\
\hline 3,4 & 0.148953 & 3,4 & 0.194100 & 3,4 & 0.139000 \\
\hline 4,1 & 0.110000 & 4,1 & 0.094300 & 4,1 & 0.110000 \\
\hline \begin{tabular}{|l|l}
4,2 & {[}
\end{tabular} & 0.157307 & 4,2 & 0.100500 & 4,2 & 0.079300 \\
\hline $4,3 \quad[$ & 0.145921 & 4,3 & 0.128300 & 4,3 & 0.083400 \\
\hline 4,4 & 0.186672 & 4,4 & 0.125300 & 4,4 & 0.130700 \\
\hline \begin{tabular}{|l|l|}
$\mathrm{T} 1,2$ \\
\end{tabular} & - & $\mathrm{T} 1,2$ & 0.001000 & $\mathrm{~T} 1,2$ & 0.001000 \\
\hline $\mathrm{T} 1,3]$ & - & $\mathrm{T} 1,3$ & 0.001000 & $\mathrm{~T} 1,3$ & 0.001200 \\
\hline $\mathrm{T1,4}$ & - & $\mathrm{T} 1,4$ & 0.098100 & $\mathrm{~T} 1,4$ & 0.126200 \\
\hline $\mathrm{T} 2,3$ & - & $\mathrm{T} 2,3$ & 0.003400 & $\mathrm{~T} 2,3$ & 0.001700 \\
\hline $\mathrm{T} 2,4$ & - & $\mathrm{T} 2,4$ & 0.039800 & $\mathrm{~T} 2,4$ & 0.055000 \\
\hline $\mathrm{T} 3,4$ & - & $\mathrm{T} 3,4$ & 0.009000 & $\mathrm{~T} 3,4$ & 0.009000 \\
\hline
\end{tabular}

\subsection{Test System 2}

This is a four-area 40 units generating system [11], with non-convexity in the cost function due to valve-point loading effect and transmission losses being neglected. Each area consists of 10 generating units and all four areas are interconnected through six tie-lines as shown in Figure 4. The figure also shows area power demands as a percentage of the total power demand (PD) of 10,500 MW. The area-wise fuel cost coefficient data may be referred from [11] and the pollutant emission coefficient data is taken from [40]. The tie-line limit from area 1 to area 2 , from area 1 to area 3 , and from area 2 to area 3 or vice versa is taken as $200 \mathrm{MW}$ and that for the remaining each tie-line is taken as $100 \mathrm{MW}$. 


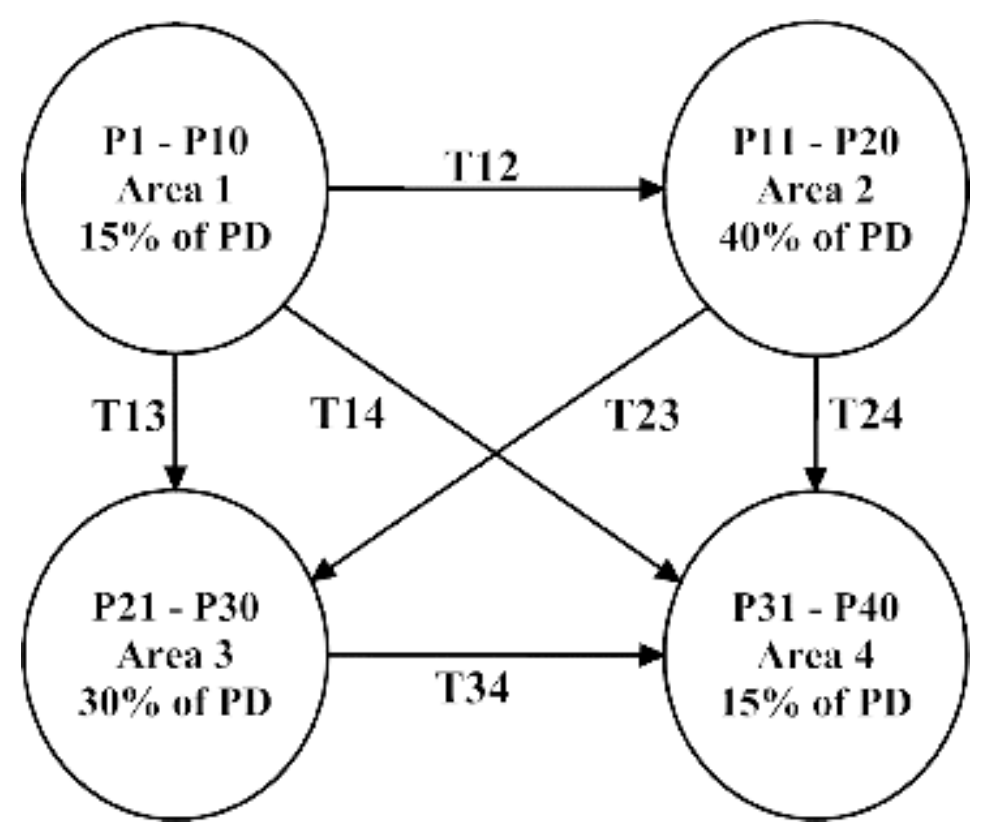

Figure 4 Four area 40 generators system for test system 2.

For case 1 and case 2, the specified spinning reserves for each area are assumed as $20 \%$ of its power demand. For case 3, the contingency spinning reserve for each area is considered as $7 \%$ of its power demand and the pooling spinning reserve is assumed as $25 \%$ of power demand of area 2. The best results obtained using EPSO for these cases are presented in Table 4. It can be observed from the table that both fuel cost and emission reduce in case 2 than in case 1 and it further reduces in case 3 . In fact, the proposed reserve sharing scheme causes a reduction of $1.154 \%$ in fuel cost and $5.132 \%$ in emission as compared with case 2 . The Pareto fronts stored in the archive for both test systems are shown in Figure 5. The figure shows very closely spaced non-dominated set of solutions for each test system. The Pareto fronts obtained are not exactly hyperbolic in shape because the MAEED problem is solved in fuzzy framework.

Table 4 Simulation results obtained using EPSO for all cases of test system 2

\begin{tabular}{|l|l|l|}
\hline Case & Fuel cost (\$/hr) & Emission (ton/hr) \\
\hline 1 & 129324.92 & 106.5239 \\
\hline 2 & 128519.35 & 87.6159 \\
\hline 3 & 127036.79 & 83.1192 \\
\hline
\end{tabular}




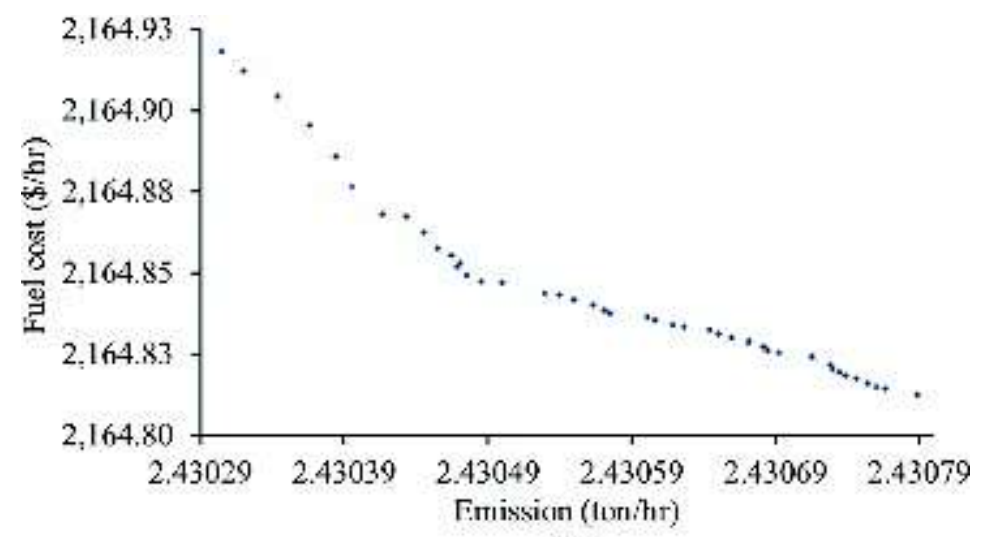

(a)

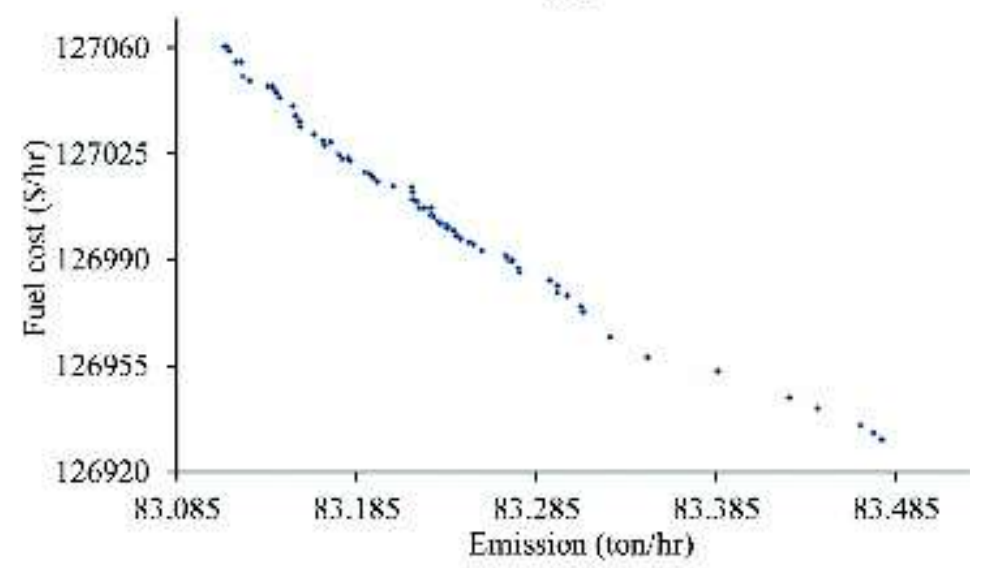

(b)

Figure 5 Pareto front for (a) Test system 1 (b) Test system 2.

Table 5 provides the contingency, pooling spinning, and available reserves for the solution obtained using EPSO. The table shows that the available reserve for each area obtained by the optimal solution is over and above than its respective contingency spinning reserve requirement. It can also be seen from the table that the sum of available reserve of all areas is maintained higher than the sum of pooling and contingency spinning reserves of all areas. The optimal generating schedule and the corresponding tie-line flows obtained for MAEED of case 3 is presented in Table 6. It can be observed from the table that the optimal solution satisfied all the problem constraints.

Table 5 Contingency, pooling spinning, and available reserves for case 3

\begin{tabular}{|l|l|l|l|l||}
\hline Reserve & Area 1 & Area 2 & Area 3 & Area 4 \\
\hline \hline Contingency spinning reserve (MW) & 110.25 & 294 & 220.5 & 110.25 \\
\hline Pooling spinning reserve (MW) & 1050 & & \\
\hline \hline Available reserve (MW) & 183.58 & 802.54 & 1060.89 & 175.00 \\
\hline
\end{tabular}


Table 6 Optimal generating schedule and tie-line flows for case 3

\begin{tabular}{|l|l|l|l|l|l||}
\hline Area, & Power & Area, & Power & Area, & Power \\
\hline \hline Unit & (MW) & Unit & (MW) & Unit & (MW) \\
\hline 1,1 & 113.9981 & 2,7 & 475.2723 & 4,3 & 177.8161 \\
\hline \hline 1,2 & 113.9987 & 2,8 & 475.8056 & 4,4 & 200.0000 \\
\hline 1,3 & 119.9993 & 2,9 & 436.8372 & 4,5 & 200.0000 \\
\hline \hline 1,4 & 179.7417 & 2,10 & 438.1827 & 4,6 & 200.0000 \\
\hline 1,5 & 96.9987 & 3,1 & 439.9741 & 4,7 & 106.6446 \\
\hline 1,6 & 136.9723 & 3,2 & 439.7863 & 4,8 & 105.8255 \\
\hline 1,7 & 299.9984 & 3,3 & 441.0615 & 4,9 & 106.9335 \\
\hline 1,8 & 299.9988 & 3,4 & 441.5426 & 4,10 & 421.6155 \\
\hline 1,9 & 299.7135 & 3,5 & 441.0398 & T1,2 & 181.5195 \\
\hline 1,10 & 130.0032 & 3,6 & 438.4807 & T1,3 & 134.9021 \\
\hline 2,1 & 318.0953 & 3,7 & 15.6718 & T1,4 & -99.9991 \\
\hline 2,2 & 317.5724 & 3,8 & 15.7586 & T2,3 & 128.9781 \\
\hline 2,3 & 402.4054 & 3,9 & 15.7953 & T2,4 & -100.0000 \\
\hline 2,4 & 394.4399 & 3,10 & 97.0000 & T3,4 & -100.0000 \\
\hline 2,5 & 394.4111 & 4,1 & 178.1622 & - & - \\
\hline 2,6 & 394.4364 & 4,2 & 178.0009 & - & - \\
\hline
\end{tabular}

From the application results of the proposed method and its comparison with existing method, it is clear that the proposed method is computationally very efficient and is capable of solving large and complex economic dispatch problems. This is due to the modifications suggested in the conventional PSO. To highlight the sequential impact of modifications suggested in inertia weight, cognitive and social behaviors of the proposed EPSO, Table 7 is presented. The table classifies ' $a$ ' as the conventional PSO, ' $b$ ' refers ' $a$ ' with exponential modulations in inertia weight, 'c' refers ' $b$ ' with preceding experience, and 'd' refers the proposed EPSO for the sake of convenience.

Table 7 Sequential modifications in the proposed EPSO

\begin{tabular}{|l|l|l|l|l||}
\hline Parameter & $\boldsymbol{a}$ & $\boldsymbol{b}$ & $\boldsymbol{c}$ \\
\hline $\mathrm{W}$ & Linear & Exponential & Exponential & Exponential \\
\hline$C_{1 b}$ & 2.0 & 2.0 & 1.6 & 1.6 \\
\hline$C_{1 p}$ & Not existing & Not existing & 0.4 & 0.4 \\
\hline$C_{2}$ & 2.0 & 2.0 & 2.0 & 2.0 \\
\hline$k_{s}$ & Not existing & Not existing & Not existing & 0.1 \\
\hline
\end{tabular}

A comparison of the set of convergence characteristics for PSO and its variants used to solve the MAEED problem for test system 2 is shown in Figure 6. This figure shows convergence for the best and average fitness, respectively. It can be observed from Figure 6(a) that subsequent modifications in the inertia weight, cognitive, and the social component in the 
control equation of the conventional PSO, the convergence characteristics are progressively improved by avoiding more and more local trappings. It can also be observed from the figure that except EPSO, the initial shape of convergence is more or less same. It happens because the constriction functions suggested in the cognitive and social components of particle's velocity play key role for better convergence in EPSO. A similar conclusion may be drawn from Figure 6(b). It can be observed from the figure that in EPSO alone, the particles do not identify the probable area of global optima during early iterations. In fact, if the promising area with global optimum is identified at the earlier stages of the optimization, there is a possibility of missing that area without exploitation [24]. Therefore, the EPSO offers advantage to exploit this region meticulously and thus converges to global or near-global optima.

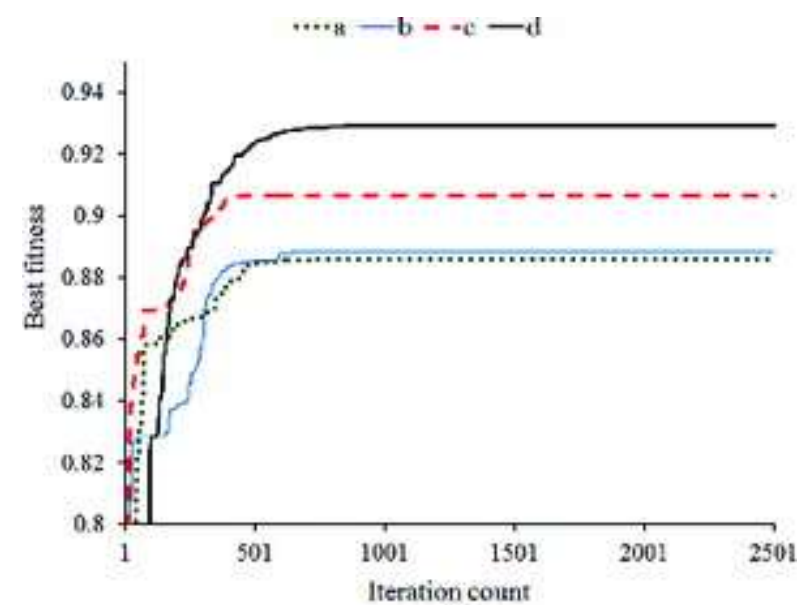

(a)

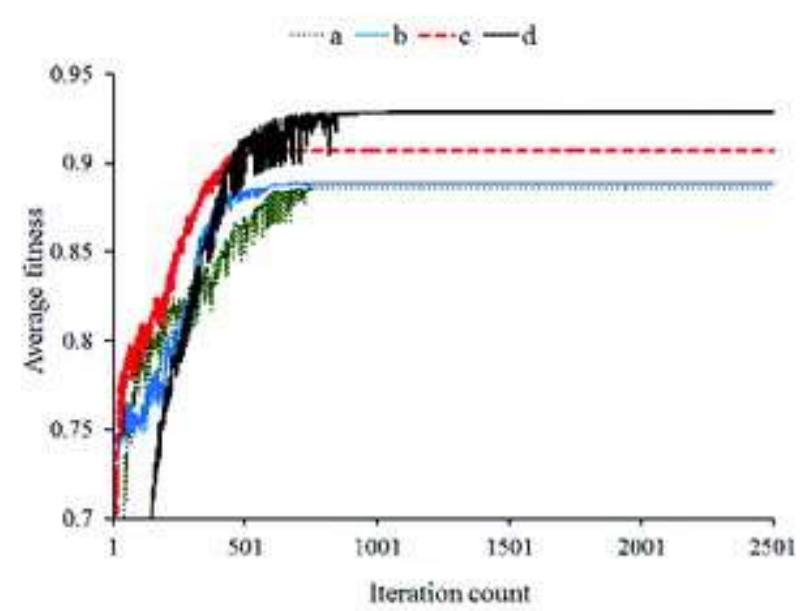

(b)

Figure 6 Convergence characteristics for (a) best fitness (b) average fitness for test system 2.

\section{Conclusions}

The MAEED problem with reserve constraint provides more economy in power generation, if area spinning reserves are also shared mutually among the interconnected areas, keeping their respective contingency spinning reserves intact. However, the system security imposes restrictions on the inter-area power transactions through tie-lines, making MAEED highly complex combinatorial constrained optimization problem. In addition, complexity arises due 
to the stringent area power balance constraints, tie-line constraints, and area reserve constraints. In addition, the cost and emission objectives of thermal plants are conflicting in nature, which further increases the complexity of the problem. In this paper, an EPSO method has been proposed to solve the complex MAEED problem. Attempts have also been made to overcome the drawbacks of the existing PSO methods by proposing EPSO method. In EPSO, the control parameters are allowed to vary with iterations in such a fashion that ensures a proper balance between cognitive and social behavior of the swarm and thus improves exploration and exploitation potentials of the PSO. This results in better convergence, higher solution quality, and stronger robustness. The application of the proposed method is investigated on two standard test generating systems with different scenarios of spinning reserve requirements for reserve sharing provisions by considering contingency and pooling spinning reserves. It has been found that the proposed reserve sharing scheme not only curtails the total reserve requirement of the system but also reduces fuel cost and pollutant emission of thermal units. The application results show that the proposed method is consistently efficient and is not trapped in local minima. It is noteworthy that EPSO does not require additional mechanism to avoid local trapping or to bound particle's velocities or squeezing the search space. Moreover, it is independent of the initial state of particles in the search space. The comparison and application results show that the proposed method is capable of producing a better solution than the other existing methods.

\section{References}

1. Sudhakar, A.V. V., Chandram, K., and Jayalaxmi, A. "Multi-area economic dispatch using secant method," J. Electr. Eng. \& Technol., Vol. 8, No. 4, pp. 708-715, 2013.

2. Sharma, M., Pandit, M., and Srivastava, L. "Reserve constrained multi-area economic dispatch employing differential evolution with time-varying mutation," Int. J. Electr. Power Energy Syst., Vol. 33, pp. 753-766, 2011.

3. Shoults, R.R., Chang, S.K., Helmick, S., and Grady, W.M. "A practical approach to unitcommitment, economic dispatch and savings allocation for multiple-area pooloperation with import/export constraints," IEEE Trans. Power Apparatus Syst., Vol. 99, No. 2, pp. 625$635,1980$.

4. Romano, R.H., Quintana, V.H., Lopez, R., and Valadez, V. "Constrained economic dispatch of multi-area systems using the Dantzig-Wolfe decomposition principle," IEEE Trans. Power Apparatus Syst., Vol. 100, No. 4, pp. 2127-2137, 1981.

5. Desell, A.L., McClelland, E.C., Tammar, K., and Horne, P.R. V. "Transmission constrained production cost analysis in power system planning," IEEE Trans. Power Apparatus Syst., Vol. 103, No. 8, pp. 2192-2198, 1984.

6. Wang, C., and Shahidehpour, S.M. "A decomposition approach to non-linear multi-area generation scheduling with tie-line constraints using expert systems," IEEE Trans. Power Syst., Vol. 7, No. 4, pp. 1409-1418, 1992.

7. Jayabarathi, T., Sadasivam, G., and Ramachandran, V. "Evolutionary programming based multi-area economic dispatch with tie line constraints," Int. J. Electr. Mach. Power Syst., Vol. 28, pp.1165-1176, 2000. 
8. Chen, C.L., and Chen, N., "Direct search method for solving economic dispatch problem considering transmission capacity constraints," IEEE Trans. Power Syst., Vol. 16, No. 4, pp. 764-769, 2001.

9. Manoharan, P.S., Kannan, P.S., Baskar, S., and Iruthayarajan, M.W. "Evolutionary algorithm solution and KKT based optimality verification to multi-area economic dispatch," Int. J. Electr. Power Energy Syst., Vol. 31, pp. 365-373, 2009.

10. Zhu, J.Z., "Multiarea power systems economic power dispatch using a nonlinear optimization neural network approach," Electr. Power Compon. and Syst., Vol. 31, No. 6, pp. $553-563,2003$.

11. Basu, M., "Artificial bee colony optimization for multi-area economic dispatch," Int. J. Electr. Power Energy Syst., Vol. 49, pp. 181-187, 2013.

12. Wang, L., and Singh, C., "Reserve-constrained multi-area environmental/economic dispatch based on particle swarm optimization with local search," Eng. Appl. Artif. Intell., Vol. 22, pp. 298-307, 2009.

13. Vlachogiannis, J.G., and Lee, K.Y., "Economic load dispatch-a comparative study on heuristic optimization techniques with an improved coordinated aggregation based PSO," IEEE Trans. Power Syst., Vol. 24, No. 2, pp. 991-1001, 2009.

14. Wang, Y., Zhou, J., Zhou, C., Wang, Y., Qin, H., and Lu, Y., "An improved self-adaptive PSO technique for short-term hydrothermal scheduling," Exp. Syst. Appl., Vol. 39, pp. 22882295, 2012.

15. Park, J.B., Jeong, Y.W., Shin, J.R., and Lee, K.Y. "An improved particle swarm optimization for non-convex economic dispatch problems,” IEEE Trans. Power Syst., Vol. 25, No. 1, pp. 156-166, February 2010.

16. Pandi, V.R., Panigrahi, B.K., Bansal, R.C., and Das, S., "Economic load dispatch using hybrid swarm intelligence based harmony search algorithm," Electr. Power Compon. Syst., Vol. 39, No. 8, pp. 751-767, 2011.

17. Zeng, Y.J., and Sun, Y., "An improved particle swarm optimization for the combined heat and power dynamic economic dispatch problem," Electr. Power Compon. Syst., Vol. 42, pp. 1700-1716, 2014.

18. Ratnaweera, A.K., Halgamuge, S.K., and Watson, H.C., "Self-organizing hierarchical particle swarm optimizer with time-varying acceleration coefficients," IEEE Trans. Evolut. Comput., Vol. 8, No. 3, pp. 240-255, June 2004.

19. Ivatloo, B.M. "Combined heat and power economic dispatch problem solution using particle swarm optimization with time varying acceleration coefficients," Electr. Power Syst. Res., Vol. 95, pp. 9-18, 2013.

20. Chalermchaiarbh, S., and Ongsakul, W., "Elitist multi-objective particle swarm optimization with fuzzy multi-attribute decision making for power dispatch," Electr. Power Compon. Syst., Vol. 40, pp. 1562-1585, 2012. 
21. Yu, B., Yuan, X., and Wang, J. "Short-term hydro-thermal scheduling using particle swarm optimization method,” Energy Convers. Manag., Vol. 48, pp. 1902-1908, 2007.

22. Wang, L., and Singh, C., "Stochastic economic emission load dispatch through a modified particle swarm optimization algorithm," Electr. Power Syst. Res., Vol. 78, pp. 1466-1476, 2008.

23. Safari, A., and Shayeghi, H., "Iteration particle swarm optimization procedure for economic load dispatch with generator constraints," Exp. Syst. Appl., Vol. 38, pp. 60436048, 2011.

24. Selvakumar, A.I., and Thanushkodi, K., "A new particle swarm optimization solution to non-convex economic dispatch problems," IEEE Trans. Power Syst., Vol. 22, No. 1, pp. 4251, February 2007.

25. Roy, R., and Ghoshal, S.P., "A novel crazy swarm optimized economic load dispatch for various types of cost functions,” Int. J. Electr. Power Energy Syst., Vol. 30, pp. 242-253, 2008.

26. Park, J.B., Lee, K.S., Shin, J.R., and Lee, K.Y., "A particle swarm optimization for economic dispatch with non-smooth cost functions," IEEE Trans. Power Syst., Vol. 20, No. 1, pp. 34-42, February 2005.

27. Barisal, A.K., "Dynamic search space squeezing strategy based intelligent algorithm solutions to economic dispatch with multiple fuels," Int. J. Electr. Power Energy Syst., Vol. 45, pp. 50-59, 2013.

28. Abido, M.A., "Multi-objective evolutionary algorithms for electric power dispatch problem," IEEE Trans. Evolut. Comput., Vol. 10, No. 3, pp. 315-329, 2006.

29. Wu, L.H., Wang, Y.N., Yuan, X.F., and Zhou, S.W., "Environmental/economic power dispatch problem using multi-objective differential evolution algorithm," Electr. Power Syst. Res., Vol. 80, pp. 1171-1181, 2010.

30. Agrawal, S., Panigrahi, B.K., and Tiwari, M.K. "Multi-objective particle swarm algorithm with fuzzy clustering for electrical power dispatch," IEEE Trans. Evolut. Comput., Vol. 12, No.5, pp. 529-541, 2008.

31. Cai, J., Ma, X., Li, Q., Li, L., and Peng, H., "A multi-objective chaotic ant swarm optimization for environmental/economic dispatch,” Int. J. Electr. Power Energy Syst., Vol. 32, pp. 337-344, 2010.

32. Kennedy, J., and Eberhart, R., Swarm Intelligence, Morgan Kaufmann Publishers, 2001.

33. Shi, Y., and Eberhart, R.C., "Empirical study of particle swarm optimization," Proc. 1999 IEEE Congress on Evolut. Comput., Piscataway, NJ, USA: IEEE Press, pp. 1945-1950.

34. Coelho, L.D. S., and Lee, C.S., "Solving economic load dispatch problems in power systems using chaotic and gaussian particle swarm optimization approaches," Int. J. Electr. Power Energy Syst., Vol. 30, pp. 297-307, 2008. 
35. Selvakumar, A.I., and Thanushkodi, K., "Optimization using civilized swarm: solution to economic dispatch with multiple minima," Electr. Power Syst. Res., Vol. 79, pp. 8-16, 2009.

36. Chaturvedi, K.T., Pandit, M., and Shrivastava, L., "Self-organizing hierarchical particle swarm optimization for non-convex economic dispatch," IEEE Trans. Power Syst., Vol. 23, No. 3, pp. 1079-1087, 2008.

37. Hayt, W.H., and Kemmerly, J.E., "Engineering Circuit Analysis," ed.,5thNew Delhi: Tata McGraw-Hill Publishing Company limited, pp. 153, 2000.

38. Ramesh, V., Jayabarathi, T., Asthana, S., Mital, S., and Basu, S., "Combined hybrid differential particle swarm optimization approach for economic dispatch problems," Electr. Power Compon. Syst., Vol. 38, 2010, pp.545-557.

39. Kuo, C.C., "A novel coding scheme for practical economic dispatch by modified particle swarm approach," IEEE Trans. Power Syst., Vol. 23, No. 4, pp 1825-1835, November 2008.

40. Basu, M., "Economic environmental dispatch using multi-objective differential evolution,” Appl. Soft Comput., Vol. 11, pp. 2845-2853, 2011.

\section{Author biographies}

Vinay Kumar Jadoun has obtained his BE (Electrical Engineering) degree in 2007 from Samrat Ashok Technological Institute (SATI), Vidisha, Madhya Pradesh, India, and M. Tech. (Power System) in 2010 from Electrical Engineering Department of Malaviya National Institute of Technology, Jaipur, India. He is currently a PhD research scholar in the Electrical Engineering Department of Malaviya National Institute of Technology, Jaipur, India. He is a Graduate Student Member of IEEE, and IEEE Power \& Energy Society (PES). His areas of interest are economic dispatch, hydrothermal scheduling, multi-area economic dispatch, soft computing techniques, and renewable power generation.

Nikhil Gupta received M. Tech. and $\mathrm{PhD}$, both in Electrical Engineering from Malaviya National Institute of Technology, Jaipur, India, in 2006 and 2012, respectively. He is presently working as Assistant Professor in the same institute. His present research interests are operation and control of power systems and computational intelligence.

Khaleequr Rehman Niazi has over 25 years of teaching and research experience. Currently, he is a Professor in the Department of Electrical Engineering, Malaviya National Institute of Technology Jaipur, India. He has published over 100 papers in journals and conferences. He has supervised several $\mathrm{PhD}$ and Master's students. He has diversified research interests in the areas of conventional power and renewable energy systems, including power system stability, distribution network reconfiguration, FACTS, and application of artificial intelligence (AI) and artificial neural network (ANN) techniques to power systems.

Anil Swarnkar received M. Tech. and PhD, both in Electrical Engineering from Malaviya National Institute of Technology, Jaipur, India, in 2005 and 2012, respectively. He is presently working as 
Assistant Professor in the same institute. His present research interests are operation and control of power systems and AI techniques.

Ramesh C. Bansal has more than 25 years of teaching, research, and industrial experience. Currently, he is the Professor and Group Head (Power) in the Department of Electrical, Electronic and Computer Engineering at the University of Pretoria, South Africa. In previous postings he was with the University of Queensland, Australia; Birla Institute of Technology and Science, Pilani, India; the University of the South Pacific, Fiji; and Civil Construction Wing, All India Radio. He has published over 200 papers in journals and at conferences. Prof. Bansal is an editor/associate editor of many reputed journals, including IET-Renewable Power Generation, IEEE access and Electric Power Components and Systems. He is a Fellow, and C Engg IET-UK, Fellow Engineers Australia, Fellow Institution of Engineers (India), and Senior Member-IEEE. He has diversified research interests in the areas of Renewable Energy and Conventional Power Systems, which includes wind, PV, hybrid power systems, distributed generation, grid integration of renewable energy, power systems analysis (reactive power/voltage control, stability, faults, and protection), smart grid, FACTS, and power quality. 